\section{Stethoscope as a Vector for Nosocomial Bacterial Infections}

Sir,

Nosocomial infection transmission contributes towards a significantly increased burden of infection diseases and carries a risk for the hospitalised inpatients, outpatients and for the healthcare workforce, via contaminated medical instruments such as a stethoscope acting as a disease transmission vector. ${ }^{1}$ Therefore, the stethoscope disinfection and hygiene maintenance practices are considered to be an important challenge for the healthcare related safety and infection control in hospitals, ${ }^{2}$ especially in the backdrop of the resurgence of the antimicrobial resistant strains, such as ciprofloxacin-resistant and gentamicin-resistant Pseudomonas aeruginosa, methicillin resistant Staphylococcus aureus (MRSA), and vancomycin resistant Enterococci (VRE). ${ }^{3}$

Sterile swabs from the diaphragm and earpieces of the medical students, residents and consultants working in different locations of The Aga Khan University Hospital, Karachi, were inoculated and cultured onto blood agar plates (BAP), chocolate agar, MacConkey agar and $\mathrm{BHI}$ (Brain heart infusion) broth and incubated at $37^{\circ} \mathrm{C}$ for 24-48 hours. Two hundred and fifty medical students, residents, physicians and consultants were also approached for their knowledge and stethoscope cleaning practices.

Just over half of the total 135 samples, i.e. $52.1 \%(n=70)$ samples, were not contaminated with bacteria, while the remaining $47.9 \% \quad(n=65)$ samples found to grow with different bacteria both commensals and pathogens. Of which, $28.2 \%(n=38)$ of the positive samples showed the growth of few colonies of coagulase negative Staphylococcus spp. (CoNS), 8\% $(n=11)$ showed moderate growth of Staphylococcus aureus; $7.9 \%(n=10)$ showed growth of Pseudomonas stutzeri, and remaining 3.8\% $(n=5)$ showed the growth of Pseudomonas aeruginosa.

These findings are supported by the earlier studies showing that coagulase negative Staphylococcus spp. (CoNS) have been the most common bacterial species isolated (up to $87.6 \%$ ) from stethoscopes. ${ }^{4}$ Although most of organisms, which got isolated in this study, are considered non-pathogenic, nevertheless, they may cause potential opportunistic infections (Ols). In Pakistan, only a handful of reports have been published about the level of awareness about the importance of stethoscope hygiene across specialties in the country, for instance, some reports say that only $38 \%$ of responders on the clinical staff have ever cleaned their stethoscopes 5 , and of those, only $53 \%$ of respondents cleaned their stethoscope by wiping with a dry cloth which has been the most commonly employed method of stethoscope cleanliness.

It calls for a frequent refrain to have efficient infection control measures along with a comprehensive educational interventions for all levels of the clinical staff. It is reported that the clinical trainees are motivated to disinfect their stethoscopes when they are made aware about the infection dissemination risks along with role modelling by senior clinicians. These findings call upon the tertiary care institutions in the country to device ways to emphasise and incorporate the regular practice of stethoscope hygiene as an important infection control strategy by incorporating instructions related to stethoscope hygiene into the medical and allied healthcare curricula.

Disclosure: This study was partially funded by the Department of Biological and Biomedical Sciences, The Aga Khan University \& Hospital Karachi.

\section{REFERENCES}

1. Rehman R, Ahmed K, Ali SM, Raza A, Rana Z. Stethoscope as vector of hospital infections. J Pak Med Assoc 2018; 68:329-30.

2. Ahmed K, Shaikh S, Rehman R, Ali SM. Frequency of awareness and practice of stethoscope hygiene with regard to guideline among medical students, residents and doctors in Karachi. JBUMDC 2018; 8:31-4.

3. Thapa S, Sapkota LB. Bacteriological assessment of stethoscopes used by healthcare workers in a tertiary care centre of Nepal. BMC Res Notes 2017; 10:353.

4. Leontsini F, Papapetropoulos A, Vantarakis A. Stethoscopes as vectors of multi-resistant coagulase negative staphylococci in a tertiary hospital. Int $J$ Med Sci Public Health 2013; 2: 324-9.

5. Hyder O. Cross-sectional study of frequency and factors associated with stethoscope cleaning among medical practitioners in Pakistan. East Mediterr Health J 2012; 18:707.

Rehana Rehman' ${ }^{1}$ Khalid Ahmed ${ }^{1}$ and Saifullah Shaikh ${ }^{2}$

1 Department of Biological and Biomedical Sciences, The Aga Khan University Hospital, Karachi, Pakistan

2 Department of Physiology, Bahria University Medical and Dental College, Karachi, Pakistan

Correspondence: Dr. Khalid Ahmed, Department of Biological and Biomedical Sciences, The Aga Khan University Hospital, Karachi, Pakistan

E-mail:khalidmd.ahmed@gmail.com

Received: July 19, 2018; Accepted: January 11, 2019 